\title{
Analysis of Channel Selection and Heterogeneous Spectrum Handoff in Cognitive Radio Ad Hoc Networks
}

\author{
Haitao Lin ${ }^{1, a}$, Gan Liu ${ }^{2, b}$ and Weimin Wü ${ }^{2, c}$ \\ ${ }^{1}$ Department of Electronics Engineering, Naval University of Engineering, Wuhan, China \\ ${ }^{2}$ Wuhan National Laboratory for Optoelectronics, Department of Electronics and Information \\ Engineering, Huazhong University of Science and Technology, Wuhan, 430074, China. \\ acclingerlisa@gmail.com, 'bliugan@hust.edu.cn(Corresponding Author), cwmwu@hust.edu.cn
}

Keywords: Cognitive radio; ad hoc networks; channel selection; channel switch; Markov-chain model; performance analysis;

\begin{abstract}
This paper addresses the priority of channel selection and switch over heterogeneous spectrum in cognitive radio ad hoc networks, which is not addressed carefully in the literature, with the aid of a novel 3-D structured Markov-chain model analysis. Our analysis indicates that the performance of the capacity, the probability of collision or switch of the Secondary Users (SUs) will be improved if the new SUs select available channels to initialize a session with a proper priority of Secondary Channels (SCs). Moreover, our work indicates that the same performance improvement would be gained if the active SUs interrupted by the PUs switch their working spectrum to available secondary channels with a proper priority. Extensive numerical analysis results verify the effectiveness of the proposed models and analysis methods.
\end{abstract}

\section{Introduction}

Compared to traditional dynamic ad hoc networks, the cognitive radio ad hoc networks (CRAHNs) achieve a higher capacity by utilizing the white hole of licensed spectrum in an opportunistic way and thus are more dynamic. This has incurred great interest in research of CRAHNs as well as presented us a great challenge to cope with its dynamic characteristics, including distributed architecture, multi-hop routing, node mobility, and dynamic switch between primary channels (PCs) and secondary channels (SCs) [1]. Originated from the initial work in [2], some works on the performance of CRAHNs focused on theoretical capacity for a CRAHN with nodes distributed in various spatial models and transmission models [3]. Other works investigated the practical performance of SUs by setting up stochastic model capturing the variation of channel state [4]. In [4], the performance is derived by the channel occupancy model in which transition probabilities are known but the state can be observed only partially. The authors of [5] studied a MAC scheme with distributed spectrum sensing and SUs' performance is evaluated by stochastic model with channel availability modeled as a two-state Markov chain. Recently, the dynamic variation of traffic of PUs and SUs is considered by the models in [6]. However, they ignored the using of SCs which can only be used by SUs but has a significant impact on licensed spectrum sharing. In [7], a Markov analysis for spectrum access in PCs for CR was presented to derive the performance of the SUs capturing the practical using of SCs. This work was improved in [8] by re-evaluating the performance of SUs under the impact of variance of heterogeneous spectrum of PCs and SCs. The authors of [8] evaluated the performance of SUs operating under PCs and SCs by a 3D Markov-chain analysis so that the traditional approximate evaluation is improved. However, they all ignore the impact of channel selection priority and switch delay on the performance of CRAHNs. For the channel switching, in the frequency range 20 $\mathrm{MHz}-8 \mathrm{GHz}$, it takes $0.5-10 \mathrm{~ms}$ to tune frequency by a step of $10 \mathrm{MHz}$ [9]. In some cognitive system [10], the sensing takes $10-50 \mathrm{~ms}$, the synchronization takes $4-18 \mathrm{~ms}$, the channel switching takes $0.5-1.5 \mathrm{~ms}$ and the channel bonding/fragmentation takes $0.5-1 \mathrm{~ms}$. For the channel selection priority, most of previous works have considered the priority of PCs for PUs, but ignored the channel selection priority for SUs which may influence the probability of channel switching and the capacity. So it is more practical for the dynamic models to consider the switch delay and channel selection priority for SUs. 
This paper addresses channel selection with the aid of a novel 3D structured Markov-chain model, considering the switch delay, which is embedded into the rate of state transition. We will illustrate that the performance of capacity as well as delay of SUs is improved for the SUs to select channels with a proper priority in comparison with the former study models without priority.

The remainder of the paper is structured as follows. In following section, we first present the system model. Then, a novel structured Markov-chain model with channel selection priority and performance analysis are provided. Afterward, numerical results and further analysis and comparison are presented. At last, conclusions are drawn in the summary section.

\section{System Model}

Both the unlicensed SCs and licensed PCs can be used by SUs subjected to the priority of PUs. For simplicity, we assume that all the CR nodes are statistically speaking identical and independent. For a certain node under consideration, the following assumptions are made:

i) The maximum numbers of PCs and SCs within the transmission range of a given node are assumed to be $c_{1}$ and $c_{2}$ respectively.

ii) The call arrival process of PUs is assumed to be Poisson with a rate of $\lambda_{1}$, while the average call holding time of PUs is assumed to be negative exponential associated with $1 / \mu_{1}$.

iii) The call arrival process of SUs is also assumed to be Poisson with a rate of $\lambda_{2}$, whose average call holding time is also assumed to be negative exponential, with an expectation of $1 / \mu_{21}$ when using PCs and $1 / \mu_{22}$ when using SCs.

iv) The variable $N_{1}(t)$ denote the number of PCs occupied by PUs, $N_{2}(t)$ the number of PCs occupied by SUs, and $N_{3}(t)$ the number of SCs occupied by SUs, at time t, respectively.

v) When a PU appcears in the channel occupied by a SU, the SU has to switch to another free channel. The SU will be dropped if no other free channel.

vi) When a new SU wants to initiates a session, it will be blocked if all the PCs and SCs are occupied, otherwise, it select a channel with or without the priority.

For a SU, the switch will happen if and only if it is preempted by a new PU, and there are other available channels for holding the interrupted SU. Note that, the transition intensity between the two states of switching will be decreased by the delay of switching. Suppose the initial transition intensity is $\lambda$, the new transition intensity is given by:

$$
\lambda_{\text {switch }}^{(1-i)}=1 /\left(\frac{1}{\lambda}+t_{\text {switch }}^{(1-i)}\right)
$$

where $t_{\text {swtch }}^{(1-i)}(\mathrm{i}=1,2)$ is the delay for a $\mathrm{SU}$ to switch from a $\mathrm{PC}$ to a $\mathrm{PC}(\mathrm{i}=1)$ or from a $\mathrm{PC}$ to a SC $(\mathrm{i}=2)$.

In v) and vi), the impact of the priority of channel selection on the performance of SUs will be studied as follows. Markov Model Performance analyses for Cannel Selection Algorithms without Priority in CRAHNs have been studied by the many works before. In this paper, we focus on the Cannel Selection Algorithms with Priority.

\section{Markov-chain Model for Channel Selection with Priority}

For simplicity, suppose a new SU as well as an active SU interrupted by a PU, always select the spare SC with a priority. Then, the state space constructed by the vector elements with three random variables $\left\{N_{1}(t), N_{2}(t), N_{3}(t)\right\}$ is a 3D Markov-Chain model. We also use SAM-HM to get the composed SSTD with $c_{1}=3, c_{2}=3$, as shown in Figure 1 . 


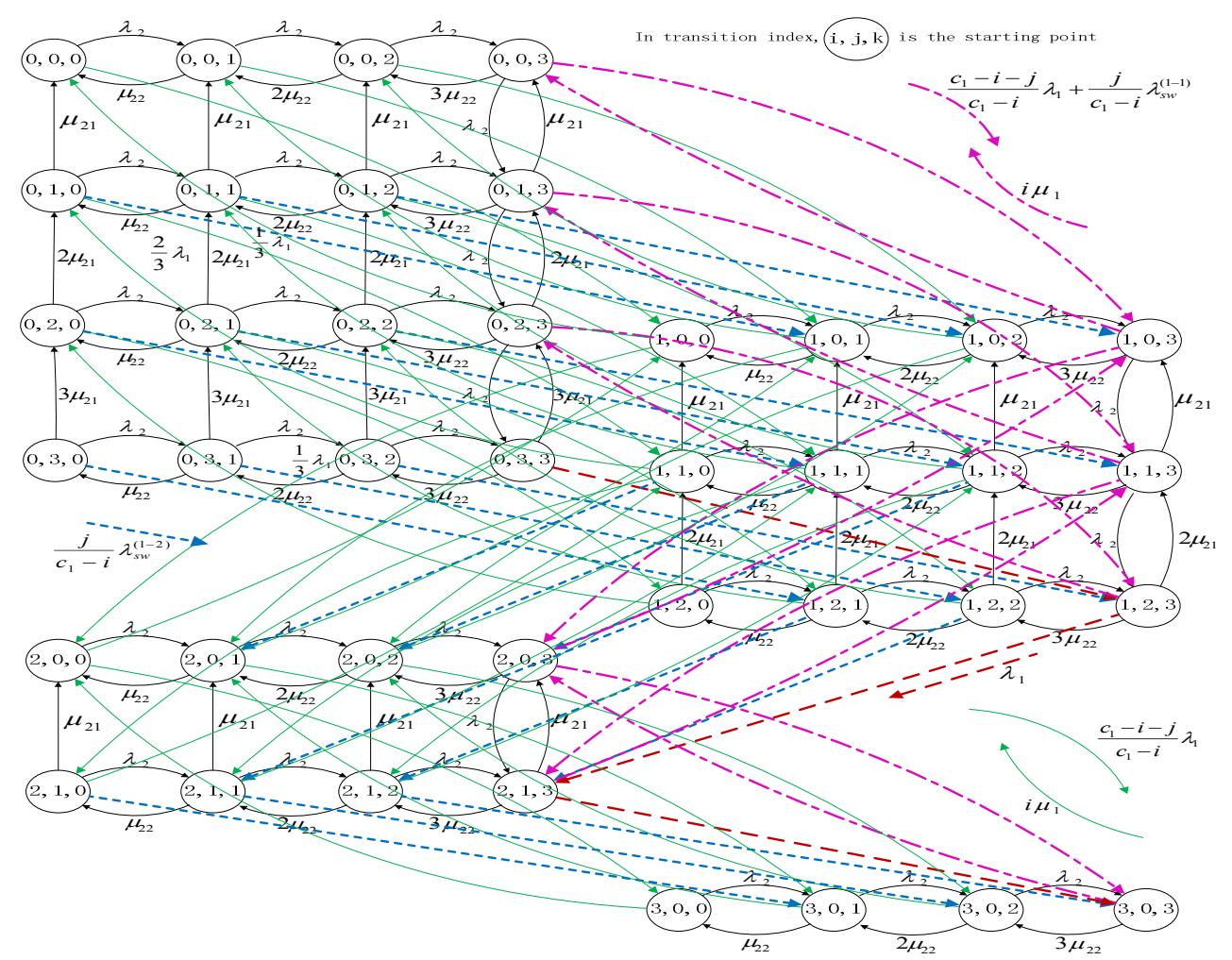

Fig.1. State Transition Diagram for Priority-Based Scheme

Similarly, the extendable equilibrium equations must be set up. At first, we consider general relationships. There are at most six relationships between the state $(i, j, k)$, in which there are spare PCs or SCs, and states $\left(i+\Delta_{i}, j+\Delta_{j}, k+\Delta_{k}\right)$, where one of $\Delta_{i}, \Delta_{j}, \Delta_{k}$ is 1 or -1 , the others equal to 0 .

Secondly, we consider the special relationships including switching or dropping.

1) When a PU initiates a session, if there are spare SCs and there are SUs using PCs, the probability for the new PU to occupy a spare PC is $\left(c_{1}-i-j\right) /\left(c_{1}-i\right)$. With a probability of $j /\left(c_{1}-i\right)$, the new PU preempt a PC occupied by a SU, which has to switch to a spare SC with a probability of 1, Therefore, the transition intensity from thce state $(i, j, k)$ to $(i+1, j, k)$ is $\lambda_{1}\left(c_{1}-i-j\right) /\left(c_{1}-i\right)$, the transition intensity from the state $(i, j, k)$ to $(i+1, j-1, k+1)$ is $\left[j /\left(c_{1}-i\right)\right] \lambda_{s w}^{(1-2)}$.

2) When a PU initiates a session, if there is no spare SC, and there are PCs occupied by SUs, the new PU will preempt a PC occcupied by a SU which has to switch to a spare PC with a transition intensity of $\frac{c_{1}-i-j}{c_{1}-i} \times \lambda_{1}+\frac{j}{c_{1}-i} \times \lambda_{s w}^{(1-1)}$ from the state $(i, j, k)$ toc, or bce droppecd if there is no spare PC with a transition intensity of $\lambda_{1}$ form the state $(i, j, k)$ (where $\left.\mathrm{j}=c_{1}-i, \mathrm{k}=c_{2}\right)$ to $(i+1, j-1, k)$.

According to the discussion above, we can set up all equilibrium equations for every state by the following general equilibrium equation with eleven undetermined coefficients $f_{m}, m=1,2, \cdots, 11$

$$
\begin{aligned}
\left(i \mu_{1}+f_{1}+j \mu_{21}+f_{2} \lambda_{2}+\right. & \left.k \mu_{22}+f_{3} \lambda_{2}\right) p_{i, j, k}= \\
& f_{4} \lambda_{1} p_{i-1, j, k}+f_{5} \lambda_{1} p_{i-1, j+1, k-1}+f_{6} \lambda_{1} p_{i-1, j+1, k}+f_{7} \mu_{1} p_{i+1, j, k} \\
+ & f_{8} \lambda_{2} p_{i, j-1, k}+f_{9} \mu_{21} p_{i, j+1, k}+f_{10} \lambda_{2} p_{i, j, k-1}+f_{11} \mu_{22} p_{i, j, k+1},
\end{aligned}
$$


where $f_{i}, i=1,2, \cdots, 11$ are determined by:

$$
\begin{aligned}
& f_{1}=\left\{\begin{array}{lc}
0 & \text { if }\left(i=c_{1}\right) \\
\frac{c_{1}-i-j}{c_{1}-i} \times \lambda_{1}+\frac{j}{c_{1}-i} \times \lambda_{s w}^{(1-2)} & \text { if }\left(i<c_{1}\right) \&\left(j \leq c_{1}-i\right) \&\left(k<c_{2}\right) \\
\frac{c_{1}-i-j}{c_{1}-i} \times \lambda_{1}+\frac{j}{c_{1}-i} \times \lambda_{s w}^{(1-1)} & \text { if }\left(i<c_{1}\right) \&\left(j<c_{1}-i\right) \&\left(k=c_{2}\right) \\
\lambda_{1} & \text { if }\left(i<c_{1}\right) \&\left(j=c_{1}-i\right) \&\left(k=c_{2}\right)
\end{array}\right. \\
& f_{2}= \begin{cases}1 & \text { if }\left(j<c_{1}-i \& \& k==c_{2}\right) \\
0 & \text { else }\end{cases} \\
& f_{3}= \begin{cases}0 & \text { if }\left(k=c_{2}\right) \\
1 & \text { else }\end{cases} \\
& f_{4}=\left\{\begin{array}{cc}
1 & \text { if }\left(i>0 \& \& k=c_{2}\right) \\
\frac{c_{1}-(i-1)-j}{c_{1}-(i-1)} & \text { if }\left(i>0 \& \& k<c_{2}\right) \\
0 & \text { else }
\end{array}\right. \\
& f_{5}= \begin{cases}\frac{j+1}{c_{1}-(i-1)} & \text { if }(i>0 \& \& k>0) \\
0 & \text { else }\end{cases} \\
& f_{6}= \begin{cases}1 & \text { if }\left(i>0 \& j=c_{1}-i \& k=c_{2}\right) \\
0 & \text { else }\end{cases} \\
& f_{7}= \begin{cases}0 & \text { if }\left(j=c_{1}-i\right) \\
i+1, & \text { else }\end{cases} \\
& f_{8}= \begin{cases}1 & \text { if }\left(j>0 \& k=c_{2}\right) \\
0 & \text { else }\end{cases} \\
& f_{9}= \begin{cases}0 & \text { if }\left(j=c_{1}-i\right) \\
j+1, & \text { else }\end{cases} \\
& f_{10}= \begin{cases}1 & \text { if }(k>0) \\
0 & \text { else }\end{cases} \\
& f_{11}= \begin{cases}0 & \text { if }\left(k=c_{2}\right) \\
k+1, & \text { else }\end{cases}
\end{aligned}
$$

The SSPD is obtained by these equations along with the following normalized condition:

$$
\sum_{i=0}^{c_{1}} \sum_{j=0}^{c_{1}-i} \sum_{k=0}^{c_{2}} p_{i, j, k}=1 .
$$

The general performance of SUs such as the blocking probability, the dropping probability and the throughput of SUs can be evaluated using the same alynasis as [7].

To start with, we can get the blocking probability of SUs:

$$
p_{\text {block }}^{(S U)}=\sum_{i=0}^{c_{1}} p_{i, c_{1}-i, c_{2}} .
$$

The dropping probability of SUs is given by:

$$
p_{d r o p}^{(S U)}=\lambda_{1} \sum_{i=0}^{c_{1}-1} p_{i, c_{1}-i, c_{2}} /\left[\lambda_{2}\left(1-p_{\text {block }}^{(S U)}\right)\right] .
$$

The throughput of SUs is given by:

$$
\text { Thoughput }^{(S U)}=\lambda_{2}\left(1-p_{\text {block }}^{(S U)}\right)\left(1-p_{\text {block }}^{(S U)}\right)
$$


We will discuss the switch probability and average delay of SUs in the following section.

Switching Probability and Delay Analysis

For the sake of evaluating the average delay of SUs, the channel switching probability of SUs has to be considered in advance. For the channel selection with priority of SCs, the probability of switching can be obtained by:

$$
\begin{aligned}
& p_{\text {switch }}^{(\text {SU })}=p_{\text {switch }}^{(1-2)}+p_{\text {switch }}^{(1-1)} \\
& \qquad=\sum_{i=0}^{c_{1}-1} \sum_{j=1}^{c_{1}-i} \sum_{k=0}^{c_{2}-1}\left(p_{i, j, k} \times \frac{j}{c_{1}-i}\right)+\sum_{i=0}^{c_{1}-1} \sum_{j=1}^{c_{1}-i-1}\left(p_{i, j, c_{2}} \times \frac{j}{c_{1}-i}\right)
\end{aligned}
$$

where $p_{\text {switch }}^{(1-i)}(\mathrm{i}=1,2)$ is the probability of switching from a PC to a PC or SC, for a SU.

For the channel selection without priority of SCs,

$$
\begin{aligned}
& p_{\text {switch }}^{(1-1)}=\sum_{i=0}^{c_{1}-1} \sum_{j=1}^{c_{1}-i-1} \sum_{k=0}^{c_{2}} p_{i, j, k} \times \frac{j}{c_{1}-i} \times \frac{c_{1}-i-j}{c_{1}+c_{2}-i-j-k} . \\
& p_{\text {switch }}^{(1-2)}=\sum_{i=0}^{c_{1}-1} \sum_{j=1}^{c_{1}-i} \sum_{k=0}^{c_{2}-1} p_{i, j, k} \times \frac{j}{c_{1}-i} \times \frac{c_{2}-k}{c_{1}+c_{2}-i-j-k} .
\end{aligned}
$$

The complex is generally $\mathrm{O}\left(n^{3}\right)$, mainly come from the solution of equations for SSPD, where $\mathrm{n}$ is the number of states: $n=\left(c_{2}+1\right)+2 \times\left(c_{2}+1\right)+\cdots+\left(c_{1}+1\right) \times\left(c_{2}+1\right)=\left(c_{2}+1\right) \frac{\left(c_{1}+1\right)^{2}+\left(c_{1}+1\right)}{2}$.

\section{Numerical Results}

In this section, we evaluate the performance of SUs by Markov model with priority in various situations by the analysis above. We investigate how the increase of arrival rate of SUs with different numbers of PCs influences the performance of SUs with other parameters as $\lambda_{1}=0.25, \mu_{1}=0.5, \mu_{2}=0.4$ users/second. and $\mu_{21}=0.3, \mu_{22}=0.5$ users/second, $t_{s w}^{(1-1)}=1 s ; t_{s w}^{(1-2)}=2 s$.

As shown in Figures 2-3, with the increase of arrival rate of SUs, the available channels for new SUs is more likely exhausted, meanwhile, the SU preempted by a new PU is less likely to switch another available channel successfully, making the increase of the blocking probability as well as the dropping probability of SUs. It is also illustrated that the larger the number of PCs is, the more available PCs for the new SUs as well as the interrupted SUs there are, and the smaller the blocking as well as the dropping probability of SUs is. Comparing the blocking and dropping probability by the two models, we can see that the result of the model with priority is lower than that without priority due to the assumption that the service rate of SUs holding by PCs is lower than that holding by SCs, i.e., $\mu_{21}<\mu_{22}$, making the average service of the SUs higher for the model with priority of SCs.

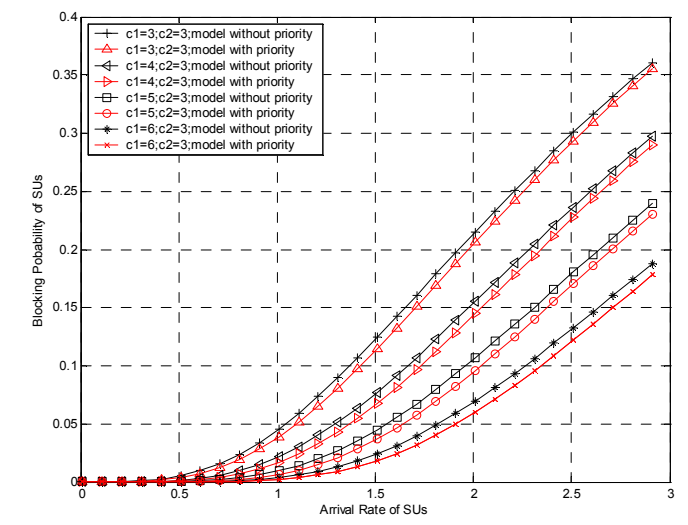

Fig.2. Blocking Probability of SUs vs. Arrival Rate of SUs.

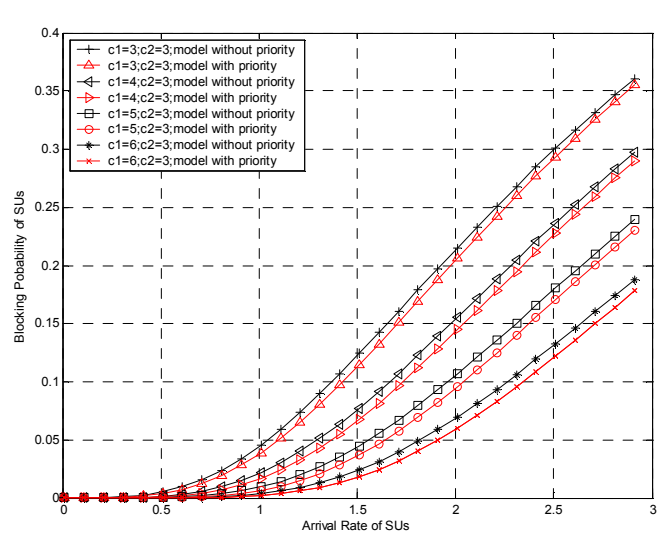

Fig.3. Dropping Probability of SUs vs. Arrival Rate of SUs. 


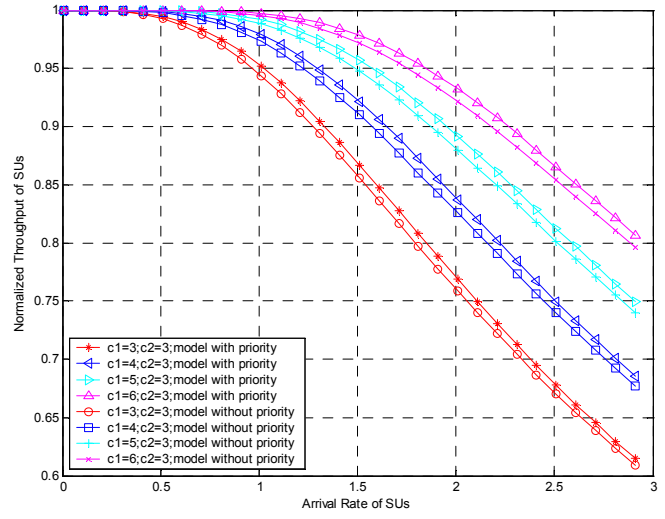

Fig.4. Normalized Throughput of SUs vs. Arrival Rate of SUs.

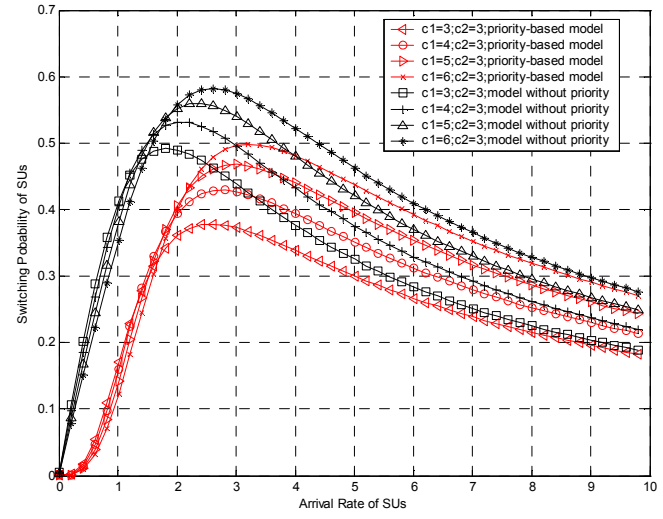

Fig.5. Switching Probability of SUs vs. Arrival Rate of PUs.

In Figure 4, with the increase of arrival rates of SUs, the available PCs and SCs for SUs decrease and thereby, the throughput of SUs decreases. Meanwhile, the larger the number of PCs, the more available PCs for SUs there are, and the higher the throughput of SUs is. In addition, the model with priority outperforms that without priority in term of the throughput of SUs due to the higher average service of the SUs. Figure 5 indicates that the switching probability of the model with priority is lower than that of the model without priority due to the less PCs occupied by SUs, who is likely to be preempted by PUs and has to switch to another available channel.

\section{Summary}

In this paper, we have investigated the impact of channel selection on the performance of the blocking probability, dropping probability and capacity as well as the switching probability. We have illustrated that the channel selection with priority of SCs will benefit the decrease of switching probability. Furthermore, if the service rates of SUs in SCs and PCs are different, then selecting the channel which can provide the SUs a higher service rate with priority will benefit the improvement of the throughput of SUs. When the SCs provide the SUs a higher or the same service rate as the PCs do, these two priorities are coincident with each other as discussed in this paper, then it is absolutely optimal for the SUs to select SCs with priority to improve its capacity and decrease the probability of spectrum handoff. Otherwise, a tradeoff should be investigated carefully in the future.

\section{Acknowledgement}

This work has been supported in part by the National Natural Science Foundation of China (No.61071068, 60972016), the China-Finnish Cooperation Project (2010DFB10570), the Funds of Distinguished Young Scientists (2009CDA150), the China Scholarship Council (CSC), as well as the National High-Tech R\&D Program of China (2006AA01Z233). 


\section{References}

[1] F. Akyildiz, W. Lee, M. C. Vuran, and S. Mohanty, "Next generation/dynamic spectrum access/cognitive radio wireless networks: A survey," Elsevier Computer Network, vol. 50, pp. 2127-2159, Sep 2006.

[2] P. Gupta and P. R. Kumar, "The capacity of wireless networks," IEEE Trans. Inf. Theory, vol.46, pp.388-404, Mar. 2000.

[3] C.X. Wang, X. Hong, and H.H. Chen, J. Thompson, “On Capacity of Cognitive Radio Networks with Average Interference Power Constraints," IEEE Trans. Wireless Commun., Vol. 8, No. 4, April 2009.

[4] Q. Zhao, L. Tong, A. Swami, and Y. Chen, "Decentralized Cognitive MAC for Opportunistic Spectrum Access in Ad Hoc Networks: A POMDP Framework," IEEE J. Sel. Areas Commun., vol. 25, no. 3, pp. 589-600, Apr. 2007.

[5] H. Su and X. Zhang, "Opportunistic MAC Protocols for Cognitive Radio Based Wireless Networks,” Proc. Ann. Conf. Information Sciences and Systems, pp. 363-368, Mar. 2007.

[6] X. Zhu, L. Shen, and T. P. Yum, "Analysis of cognitive radio spectrum access with optimal channel reservation,” IEEE Commun. Lett.,vol.11, no. 4, pp. 304-306, Apr. 2007.

[7] G. Liu, X. Zhu, and L. Hanzo, "Dynamic Spectrum Sharing Models for Cognitive Radio Aided Ad Hoc Networks and Their Performance Analysis", Proceeding of IEEE GLOBECOM 2011.

[8] G. Liu, X. Zhu, and Gx. Zhu, "Impact of Variance of Heterogeneous Spectrum on Performance of Cognitive Radio Ad Hoc Networks," Proceeding of IEEE ICC 2012.

[9] TCI 8067 Spectrum Processor Data Specification, available at http://www.tcibr.com/PDFs/8067webs.pdf, 2000.

[10]R. Chandramouli, "Cognitive Radio Networks: From Protocols to Prototypes," available at http://www.stevens.edu/spider-radio/, 2010. 